\title{
A randomized comparison of training programs using a pelvic model designed to enhance pelvic floor examination in patients presenting with chronic pelvic pain
}

\author{
Maria Giroux ${ }^{1}$ (D) Suzanne Funk ${ }^{1} \cdot$ Erwin Karreman $^{2} \cdot$ Huse Kamencic $^{1} \cdot$ Rashmi Bhargava $^{1}$
}

Received: 20 March 2020 / Accepted: 30 July 2020 / Published online: 8 September 2020

(C) The International Urogynecological Association 2020

\begin{abstract}
Introduction Pelvic floor myalgia is a common cause and contributor to chronic pelvic pain [Neurourol Urodyn 4:984-1008 (2017)]. The purpose of this study was to compare in-person versus video-based teaching methods of a comprehensive assessment of the pelvic floor musculature on a pelvic model.

Methods A randomized controlled trial of 46 participants was conducted. The participants were randomized into two groups. Both groups were taught by the same pelvic floor physiotherapist using two different teaching methods on a pelvic model. Group 1 watched an instructional video, whereas group 2 had in-person training. Both groups underwent pre- and post-training assessments consisting of a written examination and an Objective Structured Clinical Examination (OSCE). Primary outcome measure was the change in participants' pre- and post-training assessment scores. Secondary outcome measures were perceived changes in both participants' comfort level in performing pelvic floor examination and applicability of the training program to clinical practice.

Results There was no statistically significant difference between the teaching methods in the degree of improvement of the participants' mean written assessment scores $(p=0.58)$, OSCE scores $(p=0.15)$, and perceived comfort level $(p=0.19)$. Participants' mean pre- and post-assessment scores improved significantly $(p<0.001)$. Participants reported the training program to be applicable towards their clinical practice.

Conclusions This study demonstrates that learners' assessment of pelvic floor musculature can be enhanced using varied teaching methods on a pelvic model.
\end{abstract}

Keywords Chronic pelvic pain $\cdot$ Pelvic model $\cdot$ Pelvic floor musculature $\cdot$ Teaching methods $\cdot$ Video-based teaching $\cdot$ In-person teaching

Electronic Supplementary Material The video is available on https:// obgynacademy.com/chronic-pelvic-pain/. This video has been published as the monthly e-lecture at the IUGA Academy in August 2019.

Maria Giroux

mag640@mail.usask.ca

1 Department of Obstetrics and Gynecology, University of Saskatchewan, 1440 14th Avenue, Regina, Saskatchewan S4P 0W5, Canada

2 Research and Performance Support, Saskatchewan Health Authority, Regina, Saskatchewan, Canada

\author{
Abbreviations \\ ANOVA Analysis of variance \\ OSCE Objective structured clinical examination \\ PFM Pelvic floor myalgia
}

\section{Introduction}

Chronic pelvic pain is a complex multi-faceted problem that places a substantial burden on healthcare resources [1]. It is common and affects women of all ages and backgrounds. Kavvadias et al. reported that 15-20\% of women have chronic pelvic pain lasting for more than 1 year [2]. 
Pelvic floor myalgia (PFM), defined as pain originating from the pelvic floor musculature, is an important and common cause or contributor to chronic pelvic pain [3]. In a 2011 prospective cross-sectional study by Fitzgerald et al., 63\% of patients with self-reported chronic pelvic pain examined by a physician and $73.7 \%$ of patients examined by a physiotherapist were found to have PFM [4]. PFM remains a frequently unrecognized and under-treated component of chronic pelvic pain [3]. Palpation remains the best method of assessment for PFM [5]. Nonetheless, a 2011 literature review of 69 articles by Kavvadias et al. revealed that few gynecologists perform assessment of the pelvic floor musculature for the presence of myofascial pelvic pain and trigger points [4]. It is important for physicians to receive training in the comprehensive assessment of the pelvic floor musculature to identify a possible muscular cause or contribution to chronic pelvic pain and refer patients for appropriate treatment. A positive examination warrants an early referral to a pelvic floor physiotherapist. An untreated musculoskeletal component of chronic pelvic pain can result in persistent symptoms, central sensitization, subsequent patient visits to numerous health care providers, unnecessary laparoscopic surgery, psychological distress, and impaired quality of life [3].

Simulation-based training is an integral component of medical education [6]. Pelvic training models are useful tools for teaching the technique of pelvic examinations and enable learners to feel comfortable with pelvic examinations prior to patient contact [7-10].

Upon review of the literature, hands-on training was found to be superior to video-based training in teaching obstetrical emergencies and surgical skills [10-14]. Nonetheless, the 2014 study by Nilsson et al. found no significant differences in performance scores between training methods for teaching management of postpartum hemorrhage [15]. No previous studies were found that compared training methods for teaching the assessment of pelvic floor musculature in patients presenting with chronic pelvic pain.

Therefore, we designed a training program for medical students and physicians to enhance examination of pelvic floor musculature for patients presenting with chronic pelvic pain using a pelvic model. No patients were involved in the process of developing and validating the program. The objectives of the proposed training program were to identify PFM as a possible cause or contributor to chronic pelvic pain and to teach the examination of the pelvic floor musculature using a pelvic model. The purpose of this study was to compare video-based versus inperson teaching methods. We hypothesized that in-person teaching method would result in higher proficiency test scores.

\section{Materials and methods}

A randomized controlled trial was conducted between January 16 and November 19, 2018, at the Department of Obstetrics and Gynecology at the University of Saskatchewan in Regina, Saskatchewan, Canada. This quality improvement study was reviewed and exempted by the University of Saskatchewan Research Ethics Board.

Forty-six participants were assessed for eligibility. Inclusion criteria consisted of the following: $\geq 18$ years of age, learners affiliated with the College of Medicine at the University of Saskatchewan (including medical students, resident physicians in family medicine and obstetrics and gynecology programs), staff family physicians, and obstetricians and gynecologists. Informed consent was obtained from all 46 participants enrolled in the study. Exclusion criteria consisted of participants with a physical limitation that prevented them from performing a pelvic examination. None of the participants dropped out from the study. Figure 1 depicts the study flow diagram.

Participants were randomized to video $(n=23)$ and in-person $(n=23)$ groups. All participants underwent a consecutive 4$\mathrm{hr}$ training session that consisted of the following parts (Fig. 2):

\section{Part 1: Pre-training assessment}

The written examination consisted of 13 multiple choice questions, 2 short answer questions, and fill in the blanks diagrams illustrating pelvic floor musculature (Fig. 3). The 10-min OSCE station was administered by two gynecologists and consisted of a comprehensive assessment of the pelvic floor musculature on a pelvic model. The maximum score for each test was 30 points.

\section{Part 2: Educational session}

All participants viewed a 37-min video entitled "Chronic Pelvic Pain and Pelvic Floor Myalgia" and labeled diagrams of the pelvic floor anatomy to solidify their learning.

\section{Part 3: Randomization of participants into two groups}

The participants were randomized using IBM SPSS 22 software into video-based $(n=23)$ and in-person $(n=23)$ groups using stratified block randomization. Participants were stratified based on the level of training: medical students, obstetrics and gynecology resident physicians, family medicine resident physicians, staff family physicians, and obstetricians and gynecologists. After randomization, participants were allocated to different rooms. Group 1 viewed a $22-\mathrm{min}$ instructional video of a pelvic floor physiotherapist demonstrating a comprehensive assessment of the pelvic floor musculature on a pelvic model (https://www.youtube.com/watch?v= b19x9AVCUgc). The same physiotherapist delivered identical content to group 2, but with an in-person demonstration. Following the demonstration, group 2 participants were 
Fig. 1 Study flow diagram

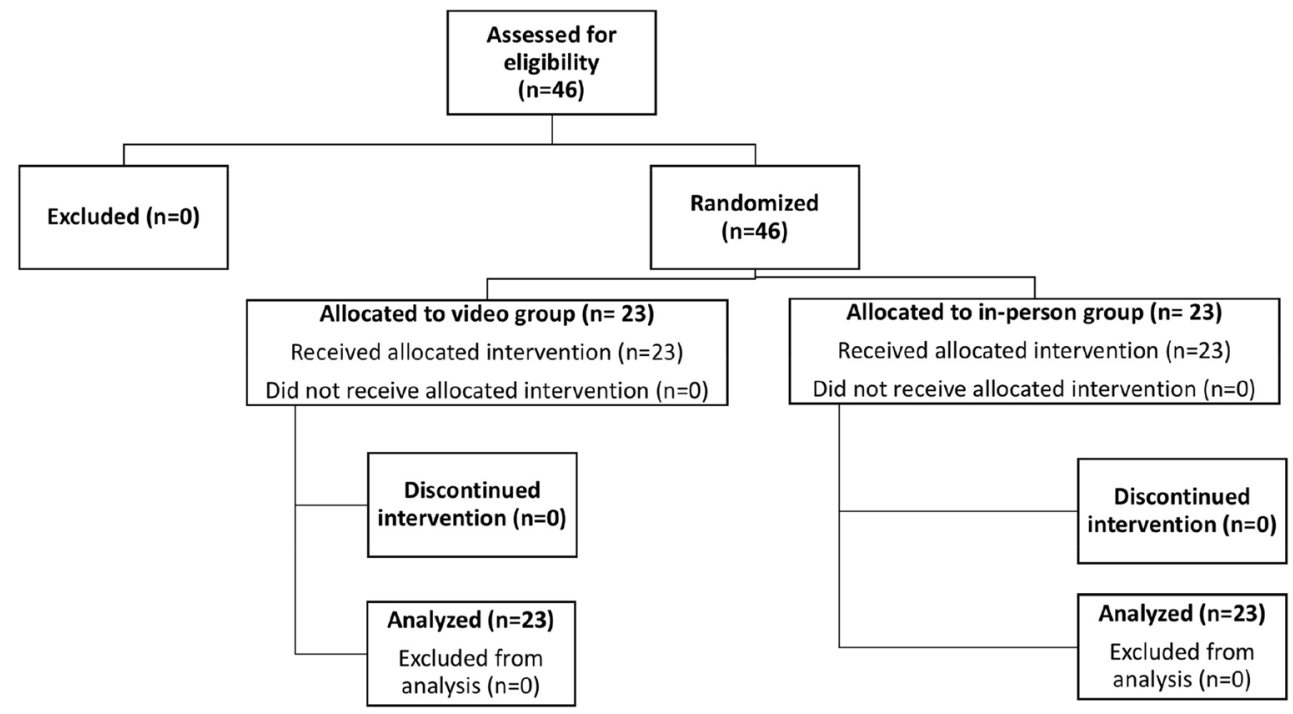

provided with immediate feedback as they assessed pelvic floor musculature on an identical pelvic model.

\section{Part 4: Post-training assessment}

Participants were re-tested using exactly the same written examination and 10-min OSCE as in the pre-training assessment. The OSCE was administered by the same two gynecologists. The evaluators were blinded to participants' group assignments and were not present at the allocation and training. All materials tested were taught in the training program.

During the training session, the use of Internet and mobile devices was prohibited, and the participants were respectfully asked to refrain from discussing content learned during the session with each other. Notes taken during the educational sessions could not be utilized during the post-training assessment. Participant feedback was obtained at the end of the session.

\section{Outcomes}

Primary outcome measure was the change in participants' preand post-training assessment scores in the written examination and OSCE. Secondary outcome measures were perceived changes in both participants' comfort level in performing pelvic floor examination and sense of applicability of the training program to clinical practice. Secondary outcome measures were assessed using a 5-point Likert-type scale.

\section{Statistical power}

Using a minimally important difference in OSCE and written assessment scores of 4.5 points (on a 30-point scale) [16], a standard deviation of 5, alpha level of 0.05 , and power of 0.80 , a sample size of 21 participants per group was required to adequately power this study (two-tailed test).
Fig. 2 Format of the training session

\section{Part 1: All participants ( $n=46$ ) had pre-training assessment (written exam and OSCE by 2 examiners)}

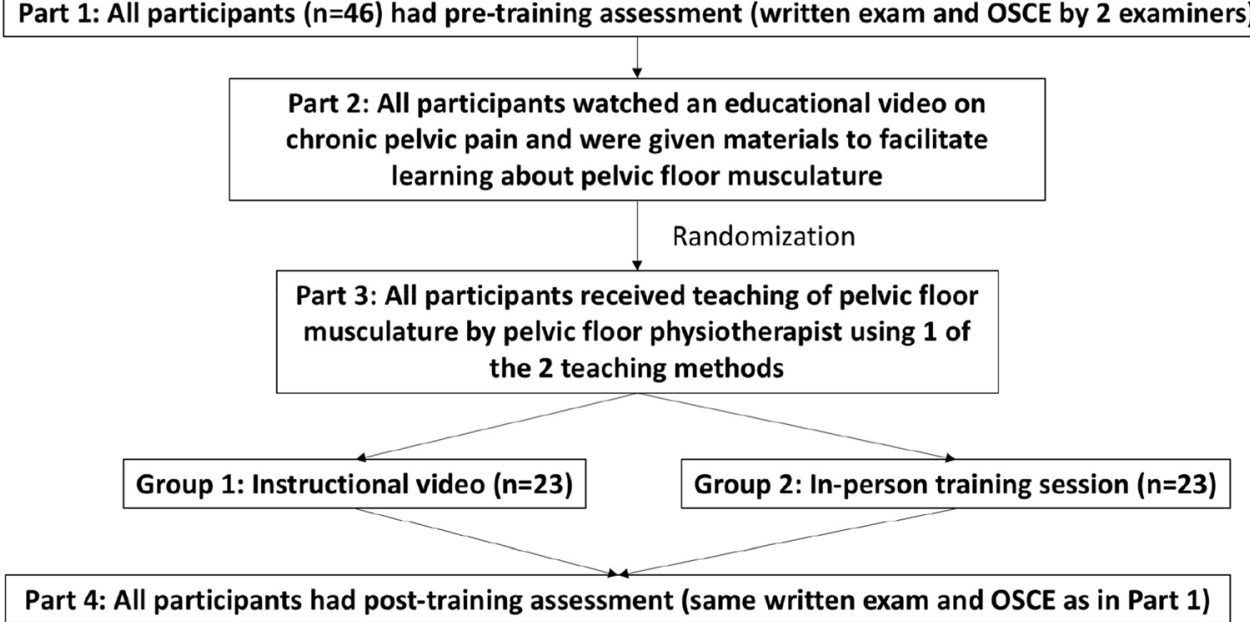


Fig. 3 Sample of the labeling exercise of pelvic floor musculature
Please colour and label the following muscles

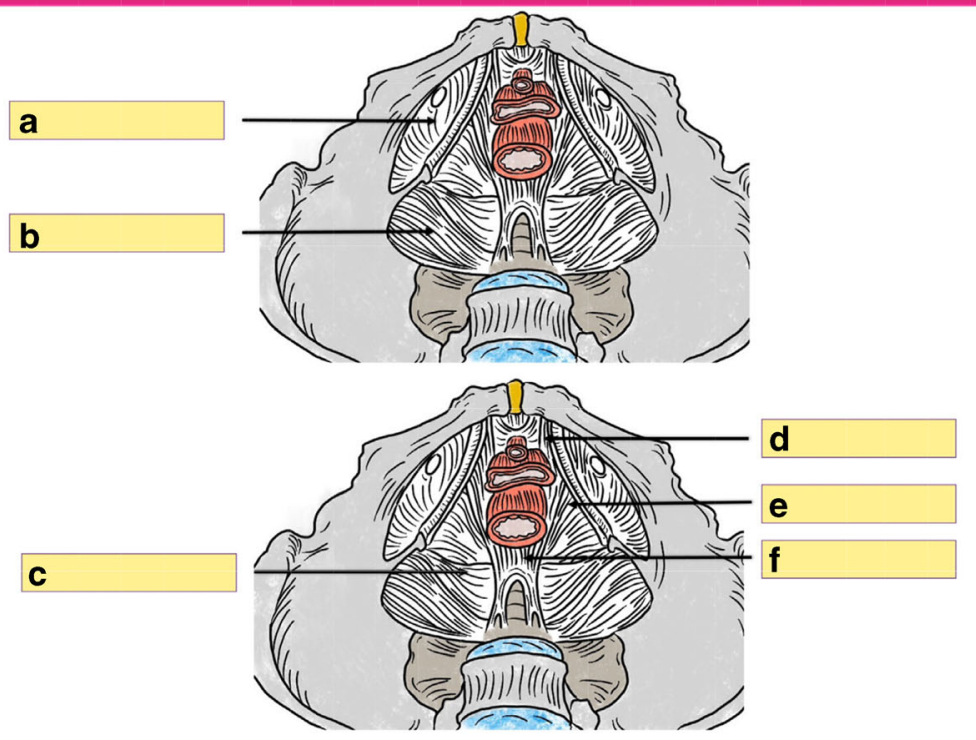

\section{Data analysis}

IBM SPSS Statistics 22 software was used for statistical analysis. Primary and secondary outcome measures were analyzed using mixed design analysis of variance (ANOVA) including main effects for group and time (pre to post). Differences in baseline characteristics between the two training groups were compared using independent t-tests for continuous variables and chi-square analyses or Fisher's exact tests for categorical variables. Any variables for which a significant difference in baseline scores between the two groups existed was accounted for by adding them as confounding variables to the main analyses to correct for their influence.

\section{Results}

Table 1 demonstrates primary and secondary outcome measures organized by group assignments. There were no significant differences in baseline characteristics between groups ( $p>0.05$ ) except for the baseline (pre-intervention) scores (Table 2). Even though participants were randomized into one of the two study groups, baseline OSCE scores were significantly higher in the in-person group $(p=0.04)$, while a similar difference approached significance for the written assessment scores $(p=0.07)$.

The mean written assessment scores improved significantly before and after training for both in-person $(p<$ $0.001)$ and video-based $(p<0.001)$ training groups. In the video group, the mean written assessment scores improved from 13.3 (95\% CI: $11.5-15.1)$ to 24.3
(95\% CI: 23.0-25.7). In the in-person group, the mean written assessment scores improved from 15.6 (95\% CI: 13.8-17.4) to 24.8 (95\% CI: 23.4-26.2) (Fig. 4). After including the baseline written assignment score as a confounding variable, there was no statistically significant difference in the degree of improvement in the mean written assessment scores between video and inperson groups $(p=0.58)$.

The mean OSCE scores also improved significantly before and after training for both in-person $(p<0.001)$ and video-based $(p<0.001)$ training groups. In the video group, the mean OSCE scores improved from 11.7 (95\% CI: $10.0-13.5)$ to 24.4 (95\% CI: 23.1-25.7). In the inperson group, the mean written assessment scores improved from 14.3 (95\% CI: $12.5-16.1)$ to 26.5 (95\% CI: 25.2-27.8) (Fig. 5). After including the baseline OSCE score as a confounding variable, there was no statistically significant difference in the degree of improvement in the mean OSCE scores between the video and in-person groups $(p=0.15)$.

The mean perceived comfort level improved significantly in both groups before and after training $(p<0.001)$. There was no statistically significant difference in the degree of improvement in perceived comfort level between the video and inperson groups $(p=0.19)$.

No significant interaction effects were found after analysis of any of the primary or secondary outcome measures, indicating that improvements in scores from pre- to post-training did not significantly differ between the two groups.

Participants reported the training program to be applicable to their clinical practice. 
Table 1 Pre- and post-training assessment scores, participants' perceived level of comfort with performing assessment of pelvic floor musculature and applicability of training program for clinical practice

$\begin{array}{lll}\text { Video group } & \text { In-person group } & \begin{array}{l}\text { Difference in the degree } \\ \text { of improvement between } \\ \text { in-person and video groups }\end{array} \\ \end{array}$

Primary outcome measures (test scores)

Written examination

Pre-training

Post-training

OSCE

Pre-training

Post-training

Secondary outcome measures

(participants' perception: maximum score out of 5)

Comfort level with performing examination

Pre-training

Post-training

Applicability of training program to clinical practice
$13.3(11.5-15.1)$

$24.3(23.0-25.7)$

$11.7(10.0-13.5)$

$24.4(23.1-25.7)$

$1.96(1.50-2.42)$

$3.43(3.09-3.78)$

$4.36(4.04-4.69)$

$\begin{array}{ll}15.6(13.8-17.4) & p=0.58 \\ 24.8(23.4-26.2) & \\ 14.3(12.5-16.1) & \\ 26.5(25.2-27.8) & \end{array}$

$26.5(25.2-27.8)$ $p=0.19$
$p=0.96$

\section{Discussion}

This study demonstrates no significant difference in the effectiveness between video-based and in-person training for teaching the assessment of the pelvic floor musculature to identify a possible muscular cause or contribution to chronic pelvic pain using a pelvic model. Improvements in written assessment scores, OSCE scores, and perceived comfort level were comparable between the video-based and in-person groups, suggesting that video-based training may be an efficient and cost-effective means to teach concepts surrounding pelvic pain to medical learners and physicians. Furthermore, video-based education can offer students several other advantages compared to more conventional means of training, including the ability to watch anytime, anywhere, to stop and rewind, and to refer back when needed [17]. The ability to effectively educate students on concepts regarding pelvic pain using video may, therefore, be highly applicable to future training of medical learners. There was no significant difference in the performance scores and degree of improvement in the perceived comfort level between training methods.

Although we hypothesized that the OSCE scores and perceived level of comfort in the in-person training group will be superior to video-based training, the results of this study disprove our hypothesis. The results of this study are different from several other studies that compared in-person versus video-based training. Studies on microsurgery, vacuumassisted delivery, shoulder dystocia management, and breech vaginal delivery, showed that hands-on learning was more effective [10-14]. Important to note, however, is that many of these skills involve complex tasks with a steep learning curve $[13,14]$. Tying surgical knots under a microscope, for instance, require meticulous practice, a high level of participant involvement and immediate feedback as well as a high degree of precision in movements, perceptual ability, and psychomotor skills [13]. The results of our study are more in line with a previous study on teaching the management for postpartum hemorrhage, a skill that, while challenging, does not involve the same level of manual dexterity that may be seen in microsurgery or the management of shoulder dystocia [15]. Therefore, despite the detailed anatomic knowledge required for evaluating myofascial trigger points for pelvic pain, it may not require the same level of previous experience, perceptual skills, or participant involvement that are required in some of the other skills listed above, which makes it more applicable to video-based learning.

Other factors likely also contributing to the success of the video include the overall quality of the video and the use of mental practice. The video, similar to the in-person training session, included the use of two anatomy models in addition to the pelvic floor model and included minor text descriptions such as "pelvic floor muscle strength" and the "modified Oxford scale for pelvic floor muscle strength," which could have helped students to stay organized in their approach to pelvic pain. Such qualities have also been endorsed in the medical education literature, namely in Dong and Goh's 2015 study regarding "Twelve tips for the effective use of videos in medical education." Tip 7, in particular, suggests that integrating PowerPoint slides, on-screen captions, and lecture images is effective because these types of information are processed in different parts of the brain [18]. Another important aspect we noted regarding the video cohort, but not the in-person training session, was that we could see participants rehearsing the sequence of the pelvic floor examination out loud or writing out steps on a piece of paper prior to post-training assessment. Mentally rehearsing steps of pelvic examination has been shown to facilitate skills acquisition and 
Table 2 Baseline characteristics of study participants. There was no statistically significant difference between the two groups for all of the listed variables $(p>0.05)$

\begin{tabular}{|c|c|c|}
\hline & $\begin{array}{l}\text { In-person group } \\
(n=23)\end{array}$ & $\begin{array}{l}\text { Video group } \\
(\mathrm{n}=23)\end{array}$ \\
\hline Mean age $( \pm \mathrm{SD})$ & $31.8( \pm 10.0)$ & $32.7( \pm 11.9)$ \\
\hline \multicolumn{3}{|l|}{ Sex } \\
\hline Male & 9 & 5 \\
\hline Female & 13 & 18 \\
\hline \multicolumn{3}{|l|}{ Handedness } \\
\hline Right-handed & 23 & 21 \\
\hline Left-handed & 0 & 2 \\
\hline Obstetricians and gynecologists & 5 & 5 \\
\hline \multicolumn{3}{|l|}{ Obstetrics and gynecology resident physicians } \\
\hline PGY1 & 1 & 1 \\
\hline PGY2 & 0 & 1 \\
\hline PGY3 & 2 & 1 \\
\hline PGY4 & 0 & 1 \\
\hline PGY5 & 1 & 1 \\
\hline Family medicine physicians & 1 & 0 \\
\hline \multicolumn{3}{|l|}{ Family medicine resident physicians } \\
\hline PGY1 & 1 & 1 \\
\hline PGY2 & 3 & 3 \\
\hline \multicolumn{3}{|l|}{ Medical students } \\
\hline Year 2 & 1 & 1 \\
\hline Year 3 & 7 & 8 \\
\hline Year 4 & 0 & 0 \\
\hline $\begin{array}{l}\text { Number of years ago performed first pelvic } \\
\text { examination in a clinical setting }\end{array}$ & Median $=3$ & Median $=3$ \\
\hline \multicolumn{3}{|l|}{$\begin{array}{l}\text { Number of patients with chronic pelvic } \\
\text { pain assess per month }\end{array}$} \\
\hline None & 10 & 9 \\
\hline$<1$ & 6 & 7 \\
\hline $1-5$ & 3 & 4 \\
\hline $5-10$ & 2 & 2 \\
\hline $10-20$ & 1 & 1 \\
\hline$>20$ & 1 & 0 \\
\hline \multicolumn{3}{|l|}{$\begin{array}{l}\text { Routinely perform assessment } \\
\text { of pelvic floor musculature in } \\
\text { patients presenting with chronic pelvic pain }\end{array}$} \\
\hline Yes & 8 & 7 \\
\hline No & 5 & 6 \\
\hline
\end{tabular}

improve performance using a pelvic model and standardized patient [19].

When assessing the applicability of our study, it is key to make note of the type of video-based learning we used. A training video, rather than in-person pelvic floor physiotherapist, was used to demonstrate the assessment to standardize the teaching experience and avoid variation in the quality of the demonstration due to inter- and intra-individual variability [20]. Nonetheless, we cannot rule out that a demonstration by a live pelvic floor physiotherapist may produce different results. In the in-person group, we used the same pelvic floor physiotherapist to demonstrate the assessment and provide immediate feedback on performance.

This study has several limitations. The first limitation of this study is that learners were educated and assessed using a pelvic model, which feels different from palpating the pelvic floor musculature of a patient. Furthermore, using a model places emphasis on technical skills, but the ability to communicate effectively and obtain informed consent are also required for clinical situations [7, 21]. Other methods of teaching, including simulated patients and anesthetized patients, also have their downfalls. While teaching pelvic examination 
Fig. 4 Mean written assessment scores before and after training in both groups (maximum score was 30)

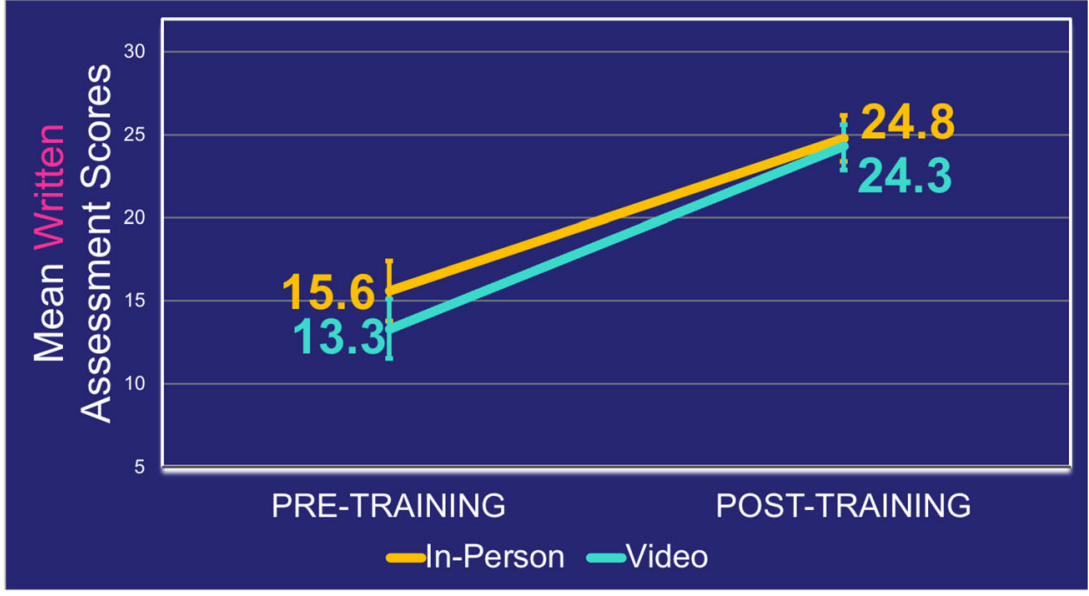

skills using standardized patients has a greater short-term improvement in clinical performance and decreases learner anxiety compared to manikin-based teaching, it is significantly more expensive, and evidence of long-term impact is lacking [21-23]. Using anesthetized patients for practicing pelvic examinations can also be useful for teaching learners, but it requires informed consent and lacks patient feedback and is not endorsed by the SOGC as the primary method of teaching pelvic examinations to medical learners [7]. Further research is required to determine whether the skills gained from this training program are transferable to the examination of the pelvic floor musculature in a clinical setting.

A second limitation is that the assessment instrument has not been validated. This imposes limitations on the overall applicability and generalizability of the study to other settings. A third limitation is that we used the same written examination and OSCE station before and after the intervention, which can result in test-enhanced learning. Lastly, we did not measure retention rates after training. We cannot exclude that the training effect may decrease over time. Due to lack of subsequent retesting, we cannot rule out that additional training sessions may be necessary for maintenance of long-term competency. Data are lacking regarding when reinforcement of training may be required. Another limitation of this study was that we did not perform a subgroup analysis to assess the effect of previous clinical experience with pelvic floor examination on the primary and secondary outcome measures.

Despite these limitations, all participants' perceived comfort level improved with training, and they found the training program can be implemented into their clinical practice. In the future, we aim to conduct a study that will compare the longterm knowledge retention between these two methods of teaching pelvic pain. These results will support whether video-based training can also produce long-term results. Future studies should also seek to create validated assessment instruments that can be used to compare different methods of teaching that allow for greater generalizability. Testing whether a combination of in-person and video-based training produces best results is also an important question to investigate.

Overall, our study supports the use of video-based training for pelvic floor myalgia and acknowledges its various strengths, including improved access to education and ability to disseminate knowledge worldwide and reach most rural and low-resource areas where in-person training may be less accessible [7]. The current pelvic floor myalgia workshop is also cost-effective, since the video is available online on the IUGA
Fig. 5 Mean OSCE scores before and after training in both groups (maximum score was 30)

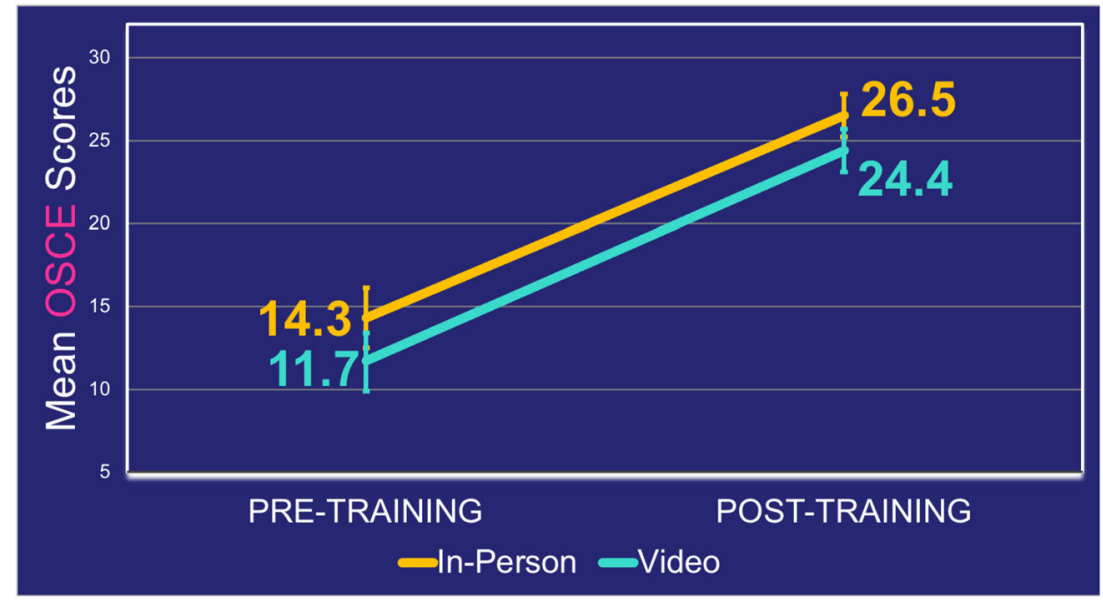


Academy, OBGYN Academy, and YouTube websites (https://obgynacademy.com/chronic-pelvic-pain/). The authors also designed a "Guide to the Assessment of the Pelvic Floor Musculature," which are cards with the anatomy of the pelvic floor and step-by-step instructions of the assessment. Healthcare providers of all levels of expertise can use the video to acquire or maintain skills at their convenience using a mobile device. This is especially useful when in-person training is not feasible, such as during the COVID19 pandemic, thus creating effective online means of knowledge dissemination is particularly important. Through this research the end goal is to disseminate knowledge to enhance care of women suffering from chronic pelvic pain through provider education.

\section{Conclusion}

This study demonstrates that learners' assessment of pelvic floor musculature can be enhanced using varied teaching methods on a pelvic model. However, there was no statistically significant difference between video-based and in-person teaching methods in degree of improvement of participants' performance and perceived comfort level with examination of the pelvic floor musculature. Participants reported the training program to be applicable towards their clinical practice.

Acknowledgements We thank the Dilawri Simulation Centre at the Regina General Hospital and Marie-Josée Forget at Total Pelvic Health for providing the pelvic models.

Author contributions Maria Giroux: Project Development, Video Preparation, Data Collection, Manuscript Preparation.

Suzanne Funk: Project Development, Video Preparation, Data Collection, Manuscript Preparation.

Huse Kamencic: Project Development, Video Preparation, Data Collection, Manuscript Preparation.

Erwin Karreman: Project Development, Data Analysis, Manuscript Preparation.

Rashmi Bhargava: Project Development, Data Collection, Manuscript Preparation.

Funding This study received funding from the Provincial Department Head Discretionary Research Fund at the University of Saskatchewan.

\section{Compliance with ethical standards}

Conflict of interest The authors declare that they have no conflict of interest.

\section{References}

1. Doggweiler R, Whitmore KE, Meijink JM, Drake MJ, Frawley H, Nordling J, et al. A standard for terminology in chronic pelvic pain syndromes: a report from the chronic pelvic pain working group of the international continence society. Neurourol Urodyn. 2017;4: 984-1008. https://doi.org/10.1002/nau.23072.

2. Kavvadias T, Baessler K, Schuessler B. Pelvic pain in urogynecology. Part 1: evaluation, definitions and diagnosis. Int Urogynecol J. 2011;22:385-93. https://doi.org/10.1007/s00192-010-1218-x.

3. Pastore EA, Katzman W. Recognizing myofascial pelvic pain in the female patient with chronic pelvic pain. J Obstet Gynecol Neonatal Nurs. 2012;41(5):680-91.

4. Fitzgerald CM, Neville CE, Mallinson T, Badillo SA, Hynes CK, $\mathrm{Tu}$ FF. Pelvic floor muscle examination in female chronic pelvic pain. J Reprod Med. 2011;56(3-4):117-22.

5. Spitznagle TM, Robinson CM. Myofascial pelvic pain. Obstet Gynecol Clin N Am. 2014;41(3):409-32.

6. Massoth C, Roder H, Ohlenburg H, Hessler M, Zarbock A, Popping D, et al. High-fidelity is not superior to low-fidelity simulation but leads to overconfidence in medical students. BMC Medical Education. 2019;19:29. https://doi.org/10.1186/s12909019-1464-7.

7. Liu K, Shapiro J, Robertson D, Chamberlain S. Pelvic examinations by medical students. J Obstet Gynaecol Can. 2010;32(99):872-4.

8. DeStephano CC, Chou B, Patel S, Rebecca S, Nancy H. A randomized controlled trial of birth simulation for medical students. Am J Obstet Gynecol. 2015;213(10):91.e1-91e7. https://doi.org/10. 1016/j.ajog.2015.03.024.

9. Edwards S, Cass G, Lenguerrand E, Fox R, Crofts J. Realism and construct validity of novel pelvic models of common gynecologic conditions. Int J Gynaecol Obstet. 2014;124(3):270-2. https://doi. org/10.1016/j.ijgo.2013.09.016.

10. Hilal Z, Kumpernatz A, Rezniczek GA, Cetin C, Tempfer-Bentz E, Tempfer C. A randomized comparison of video demonstration versus hands-on training of medical students for vacuum delivery using objective structured assessment of technical skills (OSATS). Medicine. 2017;96(11):e6355. https://doi.org/10.1097/MD. 0000000000006355.

11. Buerkle B, Pueth J, Hefler LA, Tempfer-Bentz EK, Tempfer CB. Objective structured assessment of technical skills evaluation of theoretical compared with hands-on training of shoulder dystocia management: a randomized controlled trial. Obstet Gynecol. 2012;120(4):809-14. https://doi.org/10.1097/AOG. 0b013e31826af9a9.

12. Buerke B, Rueter K, Hefler L, Tempfer-Bentz EK, Tempfer C. Objective structured assessment of technical skills (OSATS) evaluation of theoretical versus hands-on training of vaginal breech delivery management: a randomized trial. Eur J Obstet Gynecol Reprod Biol. 2013;171(2):252-6. https://doi.org/10.1016/j.ejogrb. 2013.09.015.

13. Sakamoto Y, Okamoto S, Shimzu K, Araki Y, Hirakawa A, Wakabayashi T. Hands-on simulation versus traditional videolearning in teaching microsurgery technique. Neurol Med Chir (Tokyo). 2017;57:238-45. https://doi.org/10.2176/nmc.oa.20160317.

14. Matsumoto ED, Hamstra SJ, Radomski SB, Cusimano MD. The effect of bench model fidelity on endourological skills: a randomized controlled study. J Urol. 2002;167(3):1243-7. https://doi.org/ 10.1016/S0022-5347(05)65274-3.

15. Nilsson C, Sorensen BL, Sorensen JL. Comparing hands-on and video training for postpartum hemorrhage management. Acta Obstet Gynecol Scand. 2014;93(5):517-20. https://doi.org/10. 1111/aogs.12372.

16. Jaeschke R, Singer J, Guyatt GH. Measurement of health status. Ascertaining the minimal clinically important difference control. Clin Trials. 1989;10(4):407-15.

17. Narula N, Ahmed L, Rudkowski J. An evaluation of the " 5 minute medicine" video podcast series compared to conventional medical resources for the internal medicine clerkship. Medical Teacher. 
2012;34(11):e751-5. https://doi.org/10.3109/0142159X.2012. 689446.

18. Dong C, Goh PS. Twelve tips for the effective use of videos in medical education. Medical Teacher. 2015;37(2):140-5.

19. Wartell M. Demonstrating procedures with video. Mass Transit. 1994;20(2):37-0.

20. Rakestraw PG, Irby DM, Vontver LA. The use of mental practice in pelvic examination instruction. J Med Educ. 1983;58:335-40.

21. Siwe K, Wijma K, Stjernquist M, Wijma B. Medical students learning the pelvic examination: comparison of outcome in terms of skills between a professional patient and a clinical patient model. Patient Educ Couns. 2007;68(3):211-7. https://doi.org/10.1016/j. pec.2007.05.007.

22. Janjua A, Roberts T, Okeahialam N, Clark TJ. Cost-effective analysis of teaching pelvic examination skills using Gynaecology teaching associates (GTAs) compared with manikin models (the CEAT study). BMJ Open. 2018;8:e015823. https://doi.org/10.1136/ bmjopen-2017-015823.

23. Jha V, Setna Z, Al-Hity A, Quinton ND, Roberts TE. Patient involvement in teaching and assessing intimate examination skills: a systematic review. Med Educ. 2010;44:437-357. https://doi.org/ 10.1111/j.1365-2923.2009.03609.

\section{Prior presentations:}

Video

(1) August 2019-International Urogynecological Association (IUGA) Academy-the training program selected as the monthly e-lecture.

Workshop:

(1) March 2019-Society of Obstetricians and Gynecologists of Canada (SOGC) West/Central CME (Lake Louise, AB, Canada).

Selected in the top five abstracts:

(1) April 2020-Canadian Society of Pelvic Medicine (CSPM) Annual Meeting.

\section{Podium presentations:}

(2) September 2019-American Urogynecologic Society (AUGS) and International Urogynecological Association (IUGA) Joint Scientific Meeting (Nashville, TN, US).

(3) September 2019-Canadian Society for the Advancement of Gynecologic Excellence (CanSAGE4) (Ottawa, ON, Canada).

Poster presentations:

(4) June 2019-Society of Obstetricians and Gynecologists of Canada (SOGC) Annual Clinical Scientific Conference Showcase Showdown (Halifax, NS, Canada).

(5) October 2019-International Pelvic Pain Society (IPPS) Annual Scientific Meeting (Toronto, ON, Canada).

Publisher's note Springer Nature remains neutral with regard to jurisdictional claims in published maps and institutional affiliations. 